УдК 316.65:316.662.22-057.13

\title{
КОМПАРАТИВНЫЙ АНАЛИЗ МНЕНИЙ ЛЮДЕЙ О КАРЬЕРЕ И СТАТУСЕ ПРЕДПРИНИМАТЕЛЕЙ
}

\author{
Пиньковецкая Юлия Семеновна, \\ judy54@yandex.ru \\ Ульяновский государственный университет, \\ Россия, 432000, Ульяновск, ул. Л. Толстого, 42
}

\begin{abstract}
Пиньковецкая Юлия Семеновна, кандидат экономических наук, доцент кафедры экономического анализа и государственного управления Ульяновского государственного университета.
\end{abstract}

Статья посвящена актуальной проблеме изучения мнений взрослого населения о роли предпринимателей в социально-экономической жизни современных стран. Целью исследования является оценка мнений людей о предпринимательстве в качестве хорошего варианта карьеры и статуса предпринимателя. Процесс исследования включает пять этапов. В качестве исходной информации использовались результаты опросов, проведенных в 59 странах, в процессе реализации проекта Глобального мониторинга предпринимательства. Были решены задачи оценки значений показателей, характеризующих удельные веса мужчин и женщин, считающих предпринимательство хорошей карьерой, а также высоко оценивающих социальный статус предпринимателей, в общей численности соответствующих гендерных страт. Кроме того, определялись соотношения, характеризующие сложившиеся мнения мужчин и женщин в рассматриваемых странах. Исследование основывалось на разработке экономико-математических моделей, описывающих мнения женщин и мужчин о предпринимательской карьере и высоком социальном статусе предпринимателей. Было проведено моделирование распределения шести показателей по 59 странам. По рассматриваемым странам почти две трети людей положительно относятся к предпринимательской карьере и высоко оценивают социальный статус предпринимателей. Исследование показало, что в разных странах значения удельных показателей по женщинам и мужчинам различаются незначительно, т. е. в большинстве из стран отсутствует гендерный разрыв. В 2018 г. имели место существенные различия в значениях шести рассматриваемых показателей по странам. Были определены страны, в которых наблюдались максимальные и минимальные значения шести показателей. Работа включает сопоставление значений шести показателей по России и зарубежным странам.

Ключевые слова: Предпринимательство, хорошая карьера, высокий статус, мужчины, женщины.

\section{Введение}

В последние годы предпринимательский сектор играет все более существенную роль в экономиках большинства экономически развитых и развивающихся стран. К этому сектору относится абсолютное большинство организаций в национальных экономиках, они создают около половины всех рабочих мест и обеспечивают конкуренцию на рынках товаров и услуг [1-3].

Одной из наиболее актуальных проблем развития современного предпринимательства является привлечение новых людей в создание бизнесов. Этот процесс во многом определяется тем, что, по мнению людей, предпринимательство обеспечивает хорошую карьеру и повышает социальный статус. Мнения взрослых людей о предпринимательской карьере и социальном статусе предпринимателей рассматривались в ряде исследований. Остановимся на наиболее интересных зарубежных научных публикациях. В 
статье [4] указывается, что люди воспринимают целесообразность создания и ведения собственных бизнесов под влиянием существующего в обществе социального фона. В работе [5] подчеркивается, что предпринимательство выступает в качестве совместного социального достижения людей. Социальная значимость предпринимательства и значительное влияние общественного мнения на намерения людей создать новые фирмы изучались в статье [6]. Анализ социальных предпосылок предпринимательской активности, облегчающей соответствующие процессы, рассмотрен в работе [7]. В исследованиях [8, 9] показано, что деятельность предпринимателей важна для формирования положительных социальных черт, она пользуется уважением в большинстве обществ и рассматривается в качестве хорошей карьеры для людей. В работах $[10,11]$ подчеркивается восприятие предпринимательства как деятельности, позволяющей увеличить семейный доход. Предприниматели в большей степени довольны своей карьерой и статусом по сравнению с наемными работниками [12-14].

Учитывая возрастание роли гендерных исследований в предпринимательстве [14-18], наряду с межстрановым анализом в настоящей статье рассматриваются сложившиеся различия в экспортной активности женщин и мужчин.

Необходимо отметить, что в выполненных ранее научных исследованиях проблеме гендерных особенностей мнений людей и предпринимательской карьере и социального статуса предпринимателей уделялось недостаточно внимания.

Целью исследования является оценка мнений людей, о том является ли предпринимательство хорошим выбором карьеры, а также обладают ли предприниматели высоким социальным статусом. Процесс исследования включал пять этапов. На первом этапе формировались исходные данные, описывающие мнения женщин и мужчин об их отношении к предпринимательской карьере и статусу предпринимателей по различным странам. На втором этапе оценивались значения удельных показателей, характеризующих указанные мнения людей, в общей численности этих страт населения. На третьем этапе определялись средние значения указанных показателей по рассматриваемым странам и диапазоны, в которых находятся значения этих показателей для большинства из них. На четвертом этапе проводился компаративный анализ, в процессе которого устанавливались страны, в которых отмечались минимальные и максимальные значения показателей. На пятом этапе проводился сравнительный анализ значений показателей по России и зарубежным странам.

В качестве исходной информации в исследовании использовались результаты опросов, проведенных в 59 странах, в процессе реализации проекта Глобального мониторинга предпринимательства [19]. Опросы взрослого населения (18-64 лет) в этих странах позволили получить мнения людей о восприятии ими предпринимательских возможностей и способностей.

В нашем исследовании рассматривалась оценка шести показателей, характеризующих то, как женщины и мужчины оценивали свои возможности и способности создать новый бизнес по 59 странам в 2018 году. К этим показателям относятся:

- удельный вес женщин, считающих, что предпринимательство является хорошим вариантом карьеры, в численности взрослых женщин (показатель 1);

- удельный вес мужчин, считающих, что предпринимательство является хорошим вариантом карьеры, в общей численности взрослых мужчин (показатель 2);

- отношение значений показателей, описывающих предпринимательство как хороший вариант карьеры, по женщинам и мужчинам (показатель 3);

- удельный вес женщин, считающих, что предприниматели обладают высоким социальным статусом, в численности взрослых женщин (показатель 4);

- удельный вес мужчин, считающих, что предприниматели обладают высоким социальным статусом, в общей численности взрослых мужчин, (показатель 5); 
- отношение значений показателей, характеризующих предпринимательство в качестве высокого социального статуса, по женщинам и мужчинам (показатель 6).

Проведенное исследование включало проверку пяти следующих гипотез:

- гипотеза 1 - в большинстве стран имеет место гендерный разрыв во мнениях взрослых людей о предпринимательстве в качестве хорошего варианта карьеры, т. е. мужчины и женщины по-разному относятся к своей карьере в качестве предпринимателей;

- гипотеза 2 - в большинстве стран имеет место гендерный разрыв во мнениях взрослых людей о высоком социальном статусе предпринимателей, т. е. мужчины и женщины поразному относятся к своему потенциальному статусу в качестве предпринимателей;

- гипотеза 3 - значения рассматриваемых шести показателей имеют существенную дифференциацию по разным странам;

- гипотеза 4 - территориальное расположение стран не оказывает существенного влияния на минимальные и максимальные значения каждого из шести показателей;

- гипотеза 5 - уровень доходов населения в рассматриваемых странах не оказывает существенного влияния на минимальные и максимальные значения каждого из шести показателей.

В экономико-математическом моделировании, используемом для оценки каждого из шести показателей, применялись функции нормального распределения. В статье автора [19] представлен методический подход к их разработке и использованию для определения средних значений по рассматриваемым государствам, а также диапазонов вариации значений по большинству государств. В процессе нашей работы устанавливались государства с максимальными и минимальными величинами показателей.

\section{Результаты и их обсуждение}

В процессе вычислительного эксперимента проводилось экономико-математическое моделирование на основе эмпирических данных. Модели, которые описывают распределения шести показателей по всем 59 странам, продемонстрированы далее:

- удельный вес женщин, считающих, что предпринимательство является хорошим вариантом карьеры, в численности взрослых женщин, \%:

$$
y_{1}\left(x_{1}\right)=\frac{758,57}{12,92 \times \sqrt{2 \pi}} \cdot e^{-\frac{\left(x_{1}-63,28\right)^{2}}{2 \times 12,92 \times 12,92}}
$$

- удельный вес мужчин, считающих, что предпринимательство является хорошим вариантом карьеры, в общей численности взрослых мужчин, \%:

$$
y_{2}\left(x_{2}\right)=\frac{800,71}{13,54 \times \sqrt{2 \pi}} \cdot e^{-\frac{\left(x_{2}-61,59\right)^{2}}{2 \times 13,54 \times 13,54}}
$$

- отношение значений показателей, описывающих предпринимательство как хороший вариант карьеры, по женщинам и мужчинам:

$$
y_{3}\left(x_{3}\right)=\frac{16,01}{0,10 \times \sqrt{2 \pi}} \cdot e^{-\frac{\left(x_{3}-1,07\right)^{2}}{2 \times 0,29 \times 0,29}} ;
$$

- удельный вес женщин, считающих, что предприниматели обладают высоким социальным статусом, в общей численности взрослых женщин, \%:

$$
y_{4}\left(x_{4}\right)=\frac{442,52}{9,96 \times \sqrt{2 \pi}} \cdot e^{\frac{-\left(x_{4}-69,63\right)^{2}}{2 \times 9,96 \times 9,96}} ;
$$


- удельный вес мужчин, считающих, что предприниматели обладают высоким социальным статусом, в общей численности взрослых мужчин, \%:

$$
y_{5}\left(x_{5}\right)=\frac{540,83}{10,15 \times \sqrt{2 \pi}} \cdot e^{\frac{-\left(x_{5}-69,32\right)^{2}}{2 \times 10,15 \times 10,15}}
$$

- отношение значений показателей, характеризующих предпринимательство в качестве высокого социального статуса, по женщинам и мужчинам:

$$
y_{6}\left(x_{6}\right)=\frac{11,06}{0,24 \times \sqrt{2 \pi}} \cdot e^{-\frac{\left(x_{6}-1,03\right)^{2}}{2 \times 0,24 \times 0,24}} .
$$

Высокое качество функций (1)-(6) было подтверждено в процессе тестирования по критериям Шапиро-Вилка, Пирсона и Колмогорова-Смирнова.

На следующем этапе исследования были выявлены закономерности, характеризующие сложившуюся занятость в фирмах предпринимателей-женщин и предпринимателей-мужчин, а также ее предполагаемый рост по рассматриваемым экономикам. В столбце 2 (табл. 1) приведены данные, характеризующие средние величины показателей. Диапазоны, в которых находятся значения показателей по большинству стран, продемонстрированы в третьем столбце таблицы.

\section{Таблица 1. Значения показателей, характеризующих мнения людей

\begin{tabular}{|c|c|c|}
\hline $\begin{array}{c}\text { Наименование показателя } \\
\text { Indicator }\end{array}$ & $\begin{array}{l}\text { Средние } \\
\text { величины } \\
\text { Average } \\
\text { values }\end{array}$ & $\begin{array}{c}\text { Значения } \\
\text { по большинству } \\
\text { стран } \\
\text { Values for most } \\
\text { countries }\end{array}$ \\
\hline $\begin{array}{l}\text { Удельный вес женщин, считающих, что предпринимательство является } \\
\text { хорошим вариантом карьеры, в общей численности взрослых женщин, \% } \\
\text { Share of women who believe that entrepreneurship is a good career option } \\
\text { in the total number of adult women, \% }\end{array}$ & 63,28 & $50,36-76,20$ \\
\hline $\begin{array}{l}\text { Удельный вес мужчин, считающих, что предпринимательство является } \\
\text { хорошим вариантом карьеры, в общей численности взрослых мужчин, \% } \\
\text { Share of men who believe that entrepreneurship is a good career option in } \\
\text { the total number of adult men,\% }\end{array}$ & 61,59 & $48,05-75,23$ \\
\hline $\begin{array}{l}\text { Отношение значений показателей, описывающих предприниматель- } \\
\text { ство как хороший вариант карьеры, по женщинам и мужчинам } \\
\text { Ratio of the values of indicators describing entrepreneurship as a good } \\
\text { career option for women and men }\end{array}$ & 1,07 & $0,78-1,36$ \\
\hline $\begin{array}{l}\text { Удельный вес женщин, считающих, что предприниматели обладают } \\
\text { высоким социальным статусом, в численности взрослых женщин, \% } \\
\text { Share of women who believe that entrepreneurs have a high social status in } \\
\text { the number of adult women, \% }\end{array}$ & 69,63 & $59,67-79,59$ \\
\hline $\begin{array}{l}\text { Удельный вес мужчин, считающих, что предприниматели обладают вы- } \\
\text { соким социальным статусом, в общей численности взрослых мужчин, \% } \\
\text { Share of men who believe that entrepreneurs have a high social status in } \\
\text { the total number of adult men, \% }\end{array}$ & 69,32 & $59,17-79,47$ \\
\hline $\begin{array}{l}\text { Отношение значений показателей, характеризующих предприниматель- } \\
\text { ство в качестве высокого социального статуса, по женщинам и мужчи- } \\
\text { нам } \\
\text { Ratio of the values of indicators characterizing entrepreneurship as a high } \\
\text { social status for women and men }\end{array}$ & 1,03 & $0,79-1,27$ \\
\hline
\end{tabular} Table 1. Values of indicators characterizing people's opinions}

Примечание. Расчеты проведены автором на основе функций (1)-(6).

Note. Calculations were carried out by the author based on functions (1)-(6). 


\section{Обсуждение результатов}

Среднее значение удельного веса женщин, считающих, что предпринимательство является хорошим вариантом карьеры, в общей численности взрослых женщин, достигало в 2018 г. почти 63,3 \%. Соответствующий показатель по мужчинам был несколько меньшим 61,6 \%. Таким образом, в среднем по рассматриваемым странам женщины более оптимистично относятся к предпринимательской карьере по сравнению с мужчинами. На наш взгляд, это обусловлено тем, что в большинстве развивающихся стран женщинам труднее найти хорошо оплачиваемую работу, чем мужчинам. Поэтому многие из них мечтают о создании собственных бизнесов для решения финансовых и семейных задач.

Интересно отметить, что только в десяти странах значения удельных весов женщин и мужчин, считающих предпринимательство хорошим вариантом карьеры, были примерно равны. В остальных странах имелся гендерный разрыв во мнениях по этому вопросу. В 28 странах мнение женщин о хорошем варианте карьеры предпринимателей были выше, чем у мужчин. А превышение указанного показателя по мужчинам наблюдалось в 21 стране. Таким образом, можно сделать вывод о том, что в сорока девяти странах (т. е. большинстве стран) имел место гендерный разрыв, т. е. гипотеза 1 получила свое подтверждение. Обратим внимание на то, что среднее по всем 59 рассматриваемым странам значение отношения показателей, описывающих предпринимательство как хороший вариант карьеры, по женщинам и мужчинам в 2018 г., составляло 1,07, что также подтвердило вывод о немного более оптимистичном отношении женщин к предпринимательской карьере по сравнению с мужчинами.

Среднее значение удельного веса женщин, считающих, что предприниматели обладают высоким социальным статусом, в численности взрослых женщин, достигало в 2018 г. 69,6 \%. Соответствующий показатель по мужчинам был почти на том же уровне 69,3 \%. Это подтверждает возрастание роли предпринимателей в современных экономиках, о чем было указано ранее во введении к настоящей статье. Таким образом, можно сделать вывод о высоком общественном статусе предпринимателей как в развитых, так и в развивающихся странах.

Анализ показал, что только в шести странах значения удельных весов женщин и мужчин, отмечавших в процессе опроса высокий социальный статус предпринимателей, были примерно равны. Вместе с тем в большинстве остальных стран (53) в 2018 г. отмечался гендерный разрыв. Причем, как и в случае с предыдущим показателем, имело место разнонаправленное действие этого разрыва. Интересно, что преобладающие мнения мужчин и женщин по рассматриваемым странам разделились почти поровну. Превышение значений указанных показателей по мужчинам наблюдалось в 26 странах. А в 27 странах мнение женщин о высоком общественном статусе предпринимателей было выше, чем у мужчин. Преобладание стран, в которых наблюдался гендерный разрыв, свидетельствует о том, что гипотеза 2 получила свое подтверждение. Среднее отношение значений показателей, характеризующих предпринимательство в качестве высокого социального статуса, по женщинам и мужчинам, составило в 2018 г. 1,03.

Необходимо отметить, что, по мнению, как женщин, так и мужчин высокий статус имеет несколько большее значение по сравнению с карьерными устремлениями. Это следует из сравнения значений показателей, приведенных в столбце 2 табл. 1.

Для проверки гипотезы 3 проводился анализ данных, представленных в столбце 3 табл. 1. Анализ показал существенную дифференциацию по рассматриваемым странам значений каждого из шести показателей. Следовательно, гипотеза 3 подтвердилась. Дифференциация показателей обусловлена, на наш взгляд, влиянием многих факторов, основными из которых являются: 
- исторические особенности развития предпринимательства в различных странах;

- менталитет, культурные особенности и традиции;

- уровень развития крупных концернов и корпораций со стабильной и не рисковой деятельностью;

- уровень оплаты труда и социальных гарантий в бюджетных организациях;

- сложившийся уровень безработицы;

- желание продолжить бизнесы, начатые предыдущими поколениями.

На следующем этапе выявлялись страны, в которых отмечались максимальные и минимальные значения каждого из показателей. При этом к максимальным и минимальным относятся значения, соответственно превышающие верхние границы диапазонов, продемонстрированных в третьем столбце табл. 1 и меньшие нижних границ диапазонов. Итоги этого анализа приведены в табл. 2. Наряду с перечнями стран в этой таблице представлено также подразделение выявленных стран по их географическому положению и уровню доходов населения.

\section{таблица 2. Страны с максимальными и минимальными значениями показателей} Table 2. Countries with maximum and minimum values of indicators

\begin{tabular}{|c|c|c|}
\hline $\begin{array}{c}\text { Наименование показателя } \\
\text { Indicator }\end{array}$ & $\begin{array}{c}\text { Максимальные значения } \\
\text { Maximum values }\end{array}$ & $\begin{array}{c}\text { Минимальные значения } \\
\text { Minimum values }\end{array}$ \\
\hline $\begin{array}{l}\text { Удельный вес женщин, счи- } \\
\text { тающих, что предпринима- } \\
\text { тельство является хорошим } \\
\text { вариантом карьеры, в общей } \\
\text { численности взрослых жен- } \\
\text { щин, \% } \\
\text { Share of women who believe } \\
\text { that entrepreneurship is a good } \\
\text { career option in the total num- } \\
\text { ber of adult women, \% }\end{array}$ & $\begin{array}{l}\text { США, Израиль, Гватемала, Слове- } \\
\text { ния, Китай, Великобритания, Казах- } \\
\text { стан, Швеция, Республика Корея, } \\
\text { Пуэрто-Рико. Расположены в Ев- } \\
\text { ропе три страны, в Азии - четыре, в } \\
\text { Северной Америке - одна, в Латин- } \\
\text { ской Америке - две. Доходы населе- } \\
\text { ния: высокие (семь стран), средние } \\
\text { (три страны) } \\
\text { USA, Israel, Guatemala, Slovenia, } \\
\text { China, Great Britain, Kazakhstan, } \\
\text { Sweden, Republic of Korea, Puerto } \\
\text { Rico. There are three countries in Eu- } \\
\text { rope, four countries in Asia, one coun- } \\
\text { try in North America, and two coun- } \\
\text { tries in Latin America. Population in- } \\
\text { comes: high (seven countries), me- } \\
\text { dium (three countries) }\end{array}$ & $\begin{array}{l}\text { Бразилия, Колумбия, Индия, Перу, } \\
\text { Латвия, Чили, Австрия, Ливан. } \\
\text { По две страны расположены в Ев- } \\
\text { ропе и Азии, в Латинской Америке } \\
\text { - четыре. Отмечаются высокие } \\
\text { доходы населения в трех странах, } \\
\text { средние - в трех странах, а низ- } \\
\text { кие - в двух } \\
\text { Brazil, Colombia, India, Peru, } \\
\text { Latvia, Chile, Austria, Lebanon. Two } \\
\text { countries are located in Europe and } \\
\text { Asia, in Latin America - four coun- } \\
\text { tries. High incomes of the population } \\
\text { are noted in three countries, average } \\
\text { in three countries, and low in two } \\
\text { countries }\end{array}$ \\
\hline $\begin{array}{l}\text { Удельный вес мужчин, счита- } \\
\text { ющих, что предприниматель- } \\
\text { ство является хорошим вари- } \\
\text { антом карьеры, в общей чис- } \\
\text { ленности взрослых мужчин, } \\
\% \\
\text { Share of men who believe that } \\
\text { entrepreneurship is a good ca- } \\
\text { reer option in the total number } \\
\text { of adult men, \% }\end{array}$ & $\begin{array}{l}\text { Испания, Бразилия, Колумбия, Таи- } \\
\text { ланд, Люксембург, Босния и Герце- } \\
\text { говина, Латвия, Ирландия. Располо- } \\
\text { жены в Европе пять стран, в Азии - } \\
\text { одна, в Латинской Америке - две. } \\
\text { Доходы населения высокие (четыре } \\
\text { страны), средние (три), низкие } \\
\text { (одна) } \\
\text { Spain, Brazil, Colombia, Thailand, } \\
\text { Luxembourg, Bosnia and Herze- } \\
\text { govina, Latvia, Ireland. There are five } \\
\text { countries in Europe, one country in } \\
\text { Asia, and two countries in Latin Amer- } \\
\text { ica. Population incomes are high (four } \\
\text { countries), medium (three countries), } \\
\text { low (one country) }\end{array}$ & $\begin{array}{l}\text { Индия, Канада, Ангола, Словения, } \\
\text { Марокко, Тайвань, Италия. По две } \\
\text { страны расположены в Европе, } \\
\text { Азии и Африке, одна - в Северной } \\
\text { Америке. Доходы населения высо- } \\
\text { кие (четыре страны), низкие (две) } \\
\text { India, Canada, Angola, Slovenia, } \\
\text { Morocco, Taiwan, Italy. Two coun- } \\
\text { tries are located in Europe, Asia and } \\
\text { Africa, one country in North Amer- } \\
\text { ica. Population income: high (four } \\
\text { countries), low (two countries) }\end{array}$ \\
\hline
\end{tabular}


Отношение значений показателей, описывающих предпринимательство как хороший вариант карьеры, по женщинам и мужчинам Ratio of the values of indicators describing entrepreneurship as a good career option for women and men

Удельный вес женщин, считающих, что предприниматели обладают высоким социальным статусом, в численности взрослых женщин, $\%$

Share of women who believe that entrepreneurs have a high social status in the number of adult women, $\%$

Удельный вес мужчин, считающих, что предприниматели обладают высоким социальным статусом, в общей численности взрослых мужчин, $\%$

Share of men who believe that entrepreneurs have a high social status in the total number of adult men, $\%$
Казахстан, Пуэрто-Рико, Республика Корея, Швеция, Великобритания, Ангола, Словения, Индия, Канада. По три страны расположены в Азии и Европе, по одной стране - в Африке, Латинской и Северной Америке. Доходы населения высокие (шесть стран), средние (одна), низкие (две)

Kazakhstan, Puerto Rico, Republic of Korea, Sweden, Great Britain, Angola, Slovenia, India, Canada. Three countries are located in Asia and Europe, one country each in Africa, Latin America and North America. Population income: high (six countries), medium (one country), low (two countries)

Колумбия, Гватемала, Эквадор, Польша, США, Египет, Таиланд, Панама, Китай. Расположены в Азии две страны, в Европе, Африке и Северной Америке - по одной стране, в Латинской Америке - четыре. Доходы населения высокие (три страны), средние (четыре), низкие (две)

Colombia, Guatemala, Ecuador, Poland, USA, Egypt, Thailand, Panama, China. There are two countries in Asia, one country each in Europe, Africa and North America, and four countries in Latin America. Population income: high (three countries), medium (four countries), low (two countries)

Япония, Уругвай, Швеция, Ангола, Таиланд, Кипр, Колумбия, Греция, Эквадор. В Европе и Латинской Америке расположены по три страны, в Азии - две, в Африке одна. В трех странах доходы населения высокие и средние, а низкие - в двух странах

Japan, Uruguay, Sweden, Angola, Thailand, Cyprus, Colombia, Greece, Ecuador. There are three countries in Europe and Latin America, two countries in Asia, and one country in Africa. In three countries, incomes of the population are high and medium, and low in two countries
Бразилия, Колумбия, Ирландия, Латвия, Австралия, Босния и Герцеговина, Испания. Четыре страны расположены в Европе, две - в Латинской Америке, одна - в Австралии. Высокие доходы населения имели место в четырех странах, средние доходы - в трех Brazil, Colombia, Ireland, Latvia, Australia, Bosnia and Herzegovina, Spain. Four of these countries are located in Europe, two countries in Latin America, one country in Australia. High incomes of the population took place in four countries, average incomes were in three countries

Ирландия, Индия, Мексика, Иран, Испания, Бразилия, Вьетнам, Израиль, Хорватия. Три страны расположены в Европе, четыре - в Азии, две - в Латинской Америке. Высокие доходы населения отмечаются в четырех странах, средние - в двух, низкие - в трех Ireland, India, Mexico, Iran, Spain, Brazil, Vietnam, Israel, Croatia. Three of these countries are located in Europe, four countries in Asia, two countries in Latin America. High incomes of the population are noted in four countries, average in two countries, low incomes in three countries

Франция, Марокко, Республика Корея, Канада, Российская Федерация, Чили, Перу, Индия, Австрия. Три страны расположены в Европе, по одной стране - в Африке и Северной Америке, по две - в Азии и Латинской Америке. Доходы населения высокие (пять стран), средние (две), низкие (две страны)

France, Morocco, Republic of Korea, Canada, Russian Federation, Chile, Peru, India, Austria. Three countries are located in Europe, one country each in Africa and North America, two countries each in Asia and Latin America. Population income: high (five countries), medium (two countries), low (two countries). 
Отношение значений показателей, характеризующих предпринимательство в качестве высокого социального статуса, по женщинам и мужчинам the ratio of the values of indicators characterizing entrepreneurship as a high social status for women and men
Австрия, Китай, Канада, Чили,
Польша, Российская Федерация,
Франция, Республика Корея, Ма-
рокко. В Европе расположены че-
тыре страны, в Африке, Северной и
Латинской Америке - по одной
стране, в Азии - две. Доходы насе-
ления высокие (шесть стран), сред-
ние (две), низкие (одна)
Austria, China, Canada, Chile, Poland,
Russian Federation, France, Republic
of Korea, Morocco. There are four
countries in Europe, one country each
in Africa, North and Latin America,
and two countries in Asia. Population
income: high (six countries), medium
(two countries), low (one country)

Ирландия, Мексика, Греция, Иран, Испания, Бразилия, Хорватия. Четыре страны расположены в Европе, одна - в Азии, две - в Латинской Америке. Высокие доходы населения имели место в четырех странах, средние - в двух, низкие - в одной

Ireland, Mexico, Greece, Iran, Spain, Brazil, Croatia. Four of these countries are located in Europe, one country in Asia, two countries in Latin America. High incomes of the population took place in four countries, average incomes in two countries, and in one country low incomes

Примечания. Разработано автором на основе данных табл. 1 и проекта Глобального мониторинга предпринимательства.

Note. Developed by the author based on the data in Table 1 and the Global Entrepreneurship Monitor project.

В табл. 2 приведена информация по территориальному расположению стран с максимальными (столбец 2) и минимальными (столбец 3) значениями каждого из шести оцениваемых в нашем исследовании показателей. Анализ этой информации показал, что связи между значениями показателей и территориальным расположением стран, а также уровнем доходов населения в этих странах, не наблюдается. Другими словами, страны с высокими и низкими значениями показателей расположены в различных частях света и характеризуются разным уровнем доходов населения. Таким образом, можно констатировать подтверждение гипотез 4 и 5.

Сравнительный анализ значений рассматриваемых показателей по России и зарубежным странам позволил сделать следующие выводы:

- значение показателя 1 составляет $69,9 \%$, что в 1,1 раза больше средней величины по зарубежным странам;

- значение показателя 2 составляет 53,0 \%, что в 1,2 раза меньше средней величины по зарубежным странам;

- значение показателя 3 составляет 1,3, что в 1,2 раза выше средней величины по зарубежным странам;

- значение показателя 4 составляет 74,4 \%, что в 1,1 раза выше средней величины по зарубежным странам;

- значение показателя 5 составляет 51,7 \%, что в 1,3 раза меньше средней величины по зарубежным странам;

- значение показателя 6 составляет 1,4, что в 1,4 раза больше средней величины по зарубежным странам.

Таким образом, значения удельных показателей по женщинам превышают соответствующие средние величины по зарубежным странам. Значения аналогичных показателей по мужчинам ниже в России, чем в зарубежных странах. Интересно отметить, что в России женщины более высоко, чем мужчины, оценивают как предпринимательскую карьеру, так и социальный статус предпринимателей. 


\section{Заключение}

Цель исследования, заключавшаяся в оценке мнений людей о предпринимательской карьере и предпринимательском статусе в национальных экономиках по данным за 2018 г., была достигнута. Результаты, обладающие научной новизной и оригинальностью, приведены далее:

1. Проведена оценка показателей, описывающих мнения женщин и мужчин о предпринимательской карьере и высоком социальном статусе предпринимателей.

2. Проведено моделирование распределения шести показателей по 59 странам.

3. Показано, что в различных странах значения удельных показателей по женщинам и мужчинам различаются незначительно, т. е. в большинстве из них отсутствует так называемый гендерный разрыв.

4. Доказано, что по рассматриваемым странам почти две трети людей положительно относятся к предпринимательской карьере и высоко оценивают социальный статус предпринимателей.

5. Показано, что в 2018 г. имели место существенные различия в значениях шести рассматриваемых показателей по странам.

6. Выявлены страны, для которых были характерны максимальные и минимальные значения этих шести показателей.

7. Проведено сопоставление значений показателей по России и зарубежным странам.

Итоги проведенной работы обладают определенным теоретическим и практическим значением для правительств и предпринимателей. Представленный в статье методический подход к оценке мнений людей о хорошей карьере и высоком статусе предпринимателей может применяться в дальнейших исследованиях. Полученные новые знания представляют интерес и могут использоваться в образовательном процессе в университетах.

В процессе исследования присутствовали ограничения на эмпирические данные, обусловленные тем, что рассматривалась информация только по 59 странам.

\section{СПИСОК ЛИТЕРАТУРЫ/REFERENCES}

1. Kraemer-Eis H., Lang F., Torfs W., Gvetadze S. European Small Business Finance Outlook. June 2017. EIF Working Paper 2017/43, 2017. Available at: http://www.eif.org/news_centre/research/index.htm (accessed 5 December 2020).

2. Borbás L. The role of SMEs in the European Entrepreneurship Policy. Volume of Management, Enterprise and Benchmarking in the 21st Century II. Budapest, Obuda University, Keleti Faculty of Business and Management, 2015, pp. 71-88.

3. Muller P., Julius J., Herr D., Koch L., Peycheva V., Mckiernan S. Annual report on European SMEs 2016/2017. SME Performance Review. European Union. Directorate-General for Internal Market, Industry, Entrepreneurship and SMEs. 2017. Available at: https://www.researchgate.net/publication/321268021 (accessed 20 December 2020).

4. Anderson A.R., Miller C.J. «Class matters»: human and social capital in the entrepreneurial process. Journal of Socio-Economics, 2003, vol. 1, pp. 17-36.

5. Downing S. The social construction of entrepreneurship: Narrative and dramatic processes in the coproduction of organizations and identities. Entrepreneurship: Theory \& Practice, 2005, vol. 2, pp. 185-204.

6. Rantanen T., Toikko T. Social values, societal entrepreneurship attitudes and entrepreneurial intention of young people in the Finnish welfare state. Poznan University of Economics Review, 2013, vol. 1, pp. 8-25.

7. Eckhardt J., Shane S.A. Opportunities and entrepreneurship. Journal of Management, 2003, vol. 3, pp. 333-349.

8. Padovez-Cualheta L., Borges C., Camargo A., Tavares L. An entrepreneurial career impacts on job and family satisfaction. RAUSP Management Journal, 2019, vol. 2, pp. 125-140. 
9. Kalden J., Cunningham J., Anderson A. The social status of entrepreneurs: contrasting German perspectives. The International Journal of Entrepreneurship and Innovation, 2017, vol. 18 (2), pp. 91-104. DOI: $10.1177 / 1465750317706439$.

10. Summers D. The economic impact of entrepreneurship: setting realistic expectations. Academy of Entrepreneurship Journal, 2015, vol. 21, pp. 99-107.

11. Sharma P., Sharma J. To Study the status of entrepreneurs in the Bikaner city (urban area). International Journal of Science and Research (IJSR), 2017, vol. 6 (6), pp. 2738-2740.

12. Binder M., Coad A. Life satisfaction and self-employment: a matching approach. Small Business Economics, 2013, vol. 40, pp. 1009-1033.

13. Burton M.D., Sørensen J.B., Dobrev S.D. A career perspective on entrepreneurship. Entrepreneurship Theory and Practice, 2016, vol. 40, pp. 237-247.

14. Van der Zwan P.W., Hessels J., Rietveld C.A. Self-employment and satisfaction with life, work, and leisure. Journal of Economic Psychology, 2018, vol. 64, pp. 73-88.

15. Carlianne P., Stephens H., Weinstein A. Where are all the self-employed women? Push and pull factors influencing female labor market decisions. Small Business Economics, 2016, vol. 46 (3), pp. 365-390. DOI: $10.1007 / \mathrm{s} 11187-015-9697-2$.

16. Chhabra M., Karmarkar Y. Gender gap in entrepreneurship - a study of small and micro enterprises. ZENITH International Journal of Multidisciplinary Research, 2016, vol. 6(8), pp. 82-99.

17. Sperber S., Linder C. Gender-specifics in startup strategies and the role of the entrepreneurial ecosystem. Small Business Economics, 2018, vol. 53 (4), pp. 1-14. DOI: 10.1007/s11187-018-9999-2.

18. Grosser K., Moon J. CSR and feminist organization studies: towards an integrated theorization for the analysis of gender issues. Journal of Business Ethics, 2019, vol. 155 (2), pp. 321-342. DOI: 10.1007/s10551-0173510-x.

19. Global Entrepreneurship Monitor 2018/2019. Women's Entrepreneurship Report. Global Entrepreneurship Research Association (GERA). London, United Kingdom, London Business School, 2019. 108 p.

Поступила 12.12.2020 2. Received: 12 December 2020. 
UDC 316.65:316.662.22-057.13

\title{
COMPARATIVE ANALYSIS OF PEOPLE'S OPINIONS ABOUT THE CAREER AND STATUS OF ENTREPRENEURS
}

\author{
Iuliia S. Pinkovetskaia, \\ judy54@yandex.ru \\ Ulyanovsk State University, \\ 42, L. Tolstoy street, Ulyanovsk, 432000, Russia
}

Iuliia S. Pinkovetskaia, Cand. Sc., associate professor, Ulyanovsk State University.

The article is devoted to the actual problem of studying the opinions of the adult population about the role of entrepreneurs in the socio-economic life of modern countries. The purpose of the study is to assess people's opinions about whether entrepreneurship is a good career choice, as well as whether entrepreneurs have a high social status. The research included five stages. As a starting point, the study used the results of surveys conducted in 59 countries during the implementation of the Global Entrepreneurship Monitoring project. The study solved the problems of assessing the values of indicators that characterize the proportion of men and women who consider entrepreneurship a good career, as well as highly evaluating the social status of entrepreneurs, in the total number of relevant gender strata. In addition, the relationships that characterize the prevailing opinions of men and women in the countries under consideration were determined. The study was based on the development of economic and mathematical models describing the opinions of women and men about the entrepreneurial career and high social status of entrepreneurs. We performed a simulation of the distribution of the six indicators in 59 countries. In the countries under review, almost two-thirds of people have a positive attitude to entrepreneurial careers and highly assess the social status of entrepreneurs. The study showed that in different countries, the values of specific indicators for women and men do not differ very significantly, that is, in most of them there is no gender gap. In 2018, there were significant differences in the values of the six indicators considered by country. Countries with the highest and lowest values of the six indicators were identified. The work included a comparison of the values of six indicators for Russia and foreign countries.

Key words: Entrepreneurship, good career, high status, men, women. 